\title{
Pathologie moléculaire de l'alpha 1 antitrypsine
}

L' $\alpha_{1}$ antitrypsine est capable d'inactiver des protéases à sérine ; malgré son nom, sa cible principale est l'élastase. Sa séquence compte 394 acides aminés ; son site actif est centré sur une méthionine en position 358 (voir $\mathrm{m} / \mathrm{s} n^{\circ} 2$, vol. $1, p .106$ ). Le gène qui la gouverne est localisé sur le bras long du chromosome 14. Un déficit en antitrypsine, laissant le champ libre à l'élastase, a comme conséquence majeure un emphysème pulmonaire qui survient précocément, surtout chez les fumeurs.

Deux mutants, appelés $S$ et $Z$, sont fréquents dans les populations d'origine européenne. L'activité de la molécule est normale, mais le taux dans le plasma est abaissé ; le variant $\mathrm{S}$ se retrouve à un taux de $60 \%$ de celui du type normal $M$, le variant $Z$ à $15 \%$. On admet que la limite de protection du poumon se situe à $40 \%$ de la normale. Sont donc en danger non pas les homozygotes SS (60\%) mais les homozygotes $\mathrm{ZZ}$ et, dans une moindre mesure, les hétérozygotes composites SZ (37 \%).

A l'aide d'enzymes de restriction, on a découvert dans la région du gène huit sites de polymorphisme. Dans $95 \%$ des cas, l'allèle Z est lié à un même haplotype [1]. Le variant $Z$ proviendrait donc d'une mutation unique apparue dans le nord de l'Europe à une date qui remonterait à environ 6000 ans. La fréquence du gène à l'état hétérozygote décroît en effet de $5 \%$ en Scandinavie à 1-2\% au sud de l'Europe (3\% aux USA). A l'inverse, le gène $S$ est plus fréquent au sud (10\%) qu'au nord (5\%). On admet que le maintien à un tel niveau devrait être dû à un avantage sélectif vis-à-vis d'une maladie fréquente en Europe, et on a suggéré la tuberculose. Chacun des deux variants porte une mutation ponctuelle unique affectant un acide glutami$m / s n^{\circ} 3$ ool. 3 , mors 87 que, en position 264 pour le variant $S$ (Glu $\rightarrow$ Val) et 342 pour le variant $Z$ (Glu $\rightarrow$ Lys). La perte de liaisons ioniques qui en résulte altère non pas l'activité de la molécule mais ses propriétés physicochimiques.

Le mécanisme des déficits dus aux deux variétés n'est pas le même. Le mutant $S$ possède une stabilité diminuée, notamment au cours de sa synthèse, et une partie de la protéine est dégradée dès sa naissance; de plus, la mutation crée un site d'épissage alternatif, minoritaire mais suffisant pour provoquer une perte d'ARN messager. Les effets de la mutation $\mathrm{Z}$ sont plus complexes. La production semble normale mais $85 \%$ du produit n'atteignent pas le plasma. Les ARN messagers codant pour la protéine normale et pour le variant $Z$ ont la même traductibilité et leurs produits protéiques peuvent être correctement glycosylés in vitro. $\mathrm{Si}$ on les injecte dans des ovocytes de crapaud, la synthèse initiale des deux polypeptides est la même mais, alors que toute la protéine $\mathrm{M}$ est sécrétée dans le milieu, la plus grande partie de la protéine $\mathrm{Z}$ reste bloquée dans l'ovocyte [2]. Le défaut réside donc dans la translocation de la forme $\mathrm{Z}$ à travers le réticulum endoplasmique [3]. L'antitrypsine $Z$ s'agrège pour former des inclusions visibles dans les hépatocytes; elle est ensuite protéolysée, comme en témoigne l'activité accrue des lysosomes. C'est probablement l'engorgement des hépatocytes qui est responsable de l'atteinte hépatique fréquemment associée au déficit homozygote $\mathrm{ZZ}$.

L'utilisation thérapeutique de $l^{\prime} \alpha_{1}$ antitrypsine a été envisagée pour la lutte contre l'emphysème. $\mathrm{m} / \mathrm{s}$ a déjà mentionné que la présence au niveau du centre actif d'une méthionine, facilement oxydable, rendait la protéine fragile, d'où l'intérêt de son remplace- ment par une valine, non oxydable et qui n'altère pas les propriétés catalytiques. Une application nouvelle et passionnante de ces possibilités a été récemment découverte [3] : le syndrome de choc est en relation avec une activation des polynucléaires neutrophiles, accompagnée de libération massive d'élastase et d'autres protéases, qui aboutit à l'inactivation de l'antitrypsine et des autres antiprotéases. Les mutants stables de l'antitrypsine pourraient être utilisés dans la prophylaxie du syndrome de choc. Enfin, un mutant naturel extraordinaire, que nous avions également cité, est le mutant Pittsburgh dans lequel la méthionine 358 est remplacée par une arginine; il a perdu les propriétés de l'antitrypsine en acquérant celles d'une antithrombine. Or, cette mutation, qui a pu être reproduite artificiellement, confere également à la molécule une activité antikallikréine; elle inhibe l'ensemble des "protéines du système de contact ", qui déclenchent la cascade de coagulation au contact des plaquettes et qui contribuent à la gravité du syndrome de choc [4]. Les antitrypsines mutées que pourra produire le génie génétique devraient donc fournir tout un arsenal thérapeutique utilisable dans la prévention de l'emphysème comme dans la prévention et le traitement des états de choc. J.-C. D.

1. Cox DW, Woo SLC, Mansfield T. DNA restriction fragments associated with $\alpha_{1}$ antitrypsin indicate a single origin for deficiency allele PIZ. Nature 1985 ; 316 : 79-81. 2. Perlmutter DH, Kay RM, Cole FS, et al. The cellular defect in proteinase inhibitor deficiency is expressed in human monocytes and in Xenopus oocytes injected with human liver mRNA. Proc Natl Acad Sci USA 1985 ; 82 . 6918-21.

3. Carrell RW. $\alpha_{1}$ antitrypsin : molecular pathology, leucocytes, and tissue damage. $J$ Clin Invest $1986 ; 78$ : 1427-31.

4. Scott CF, Carrell RW, Glaser CH, et al. $\alpha_{1}$ antitrypsin Pittsburg. A potent inhibitor of human plasma factor XIa, Kallikrein and factor XIIf. J Clin Invest 1986 ; 77 : 631-4.
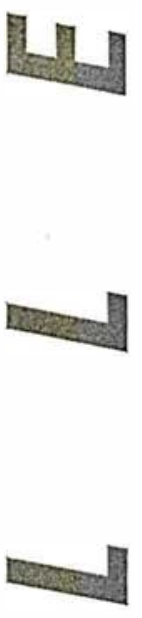

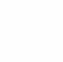
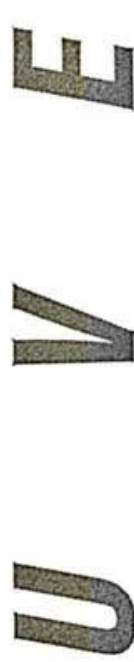